\title{
Impacts of emission changes in China from 2010 to 2017 on domestic and intercontinental air quality and health effect
}

Yuqiang Zhang ${ }^{1}$, Drew Shindell ${ }^{1,2,3}$, Karl Seltzer ${ }^{1}$, Lu Shen ${ }^{4}$, Jean-Francois Lamarque ${ }^{5}$, Qiang Zhang $^{6}$, Bo Zheng ${ }^{7}$, Jia Xing ${ }^{8}$, Zhe Jiang ${ }^{9}$, Lei Zhang ${ }^{10}$

Supporting information

Fig S1. The anthropogenic emission trends in China from 2000 to 2017 reported by MEIC and CEDS. Note that the CEDS did not report emissions after year 2014 (Hoesly et al., 2018).
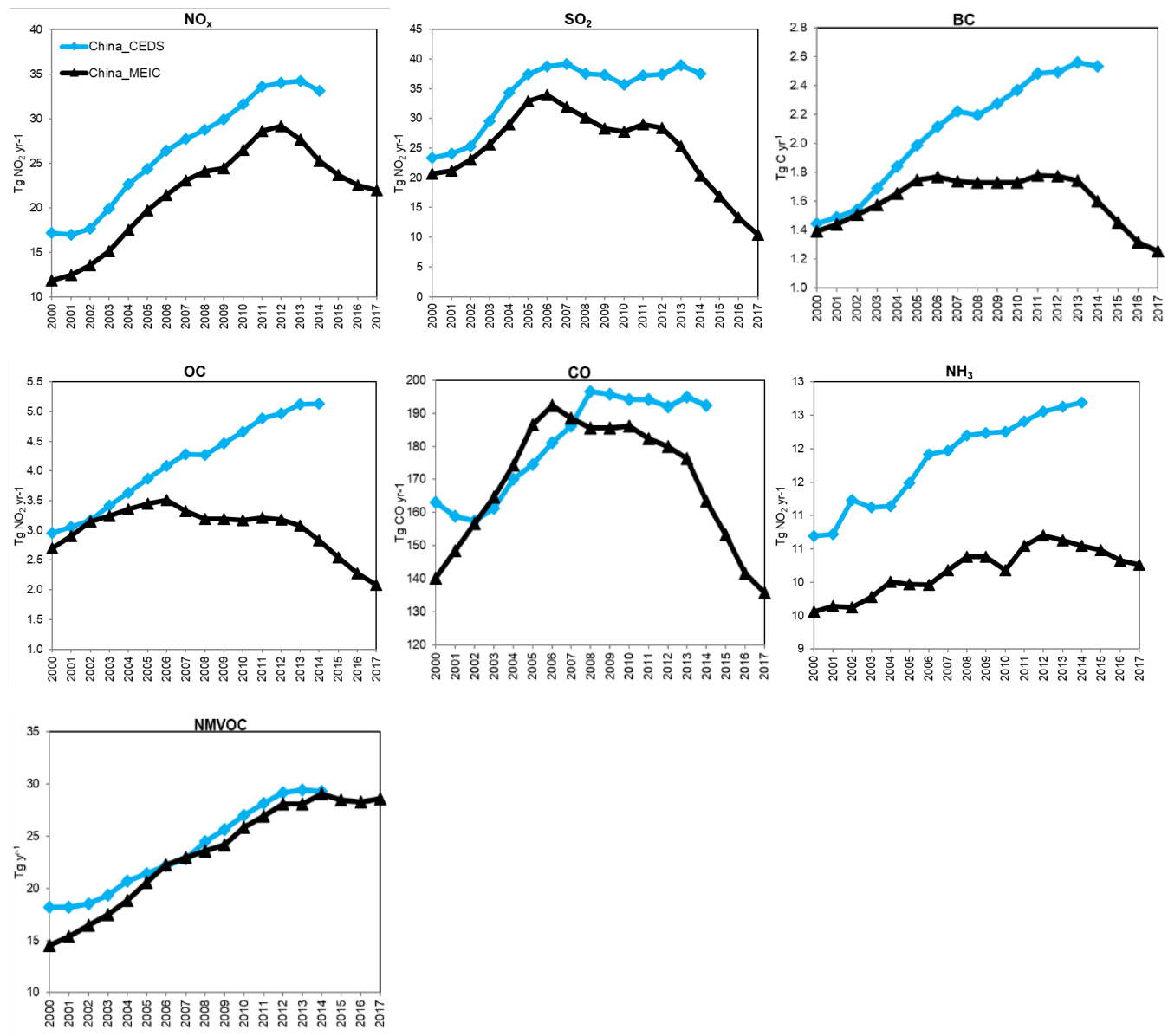
Fig S2. The spatial distribution for emission relative differences between CEDS and MEIC in 2014 for (a) $\mathrm{NO}_{\mathrm{x}}$, (b) $\mathrm{SO}_{2}$, (c) BC, (d) OC and (e) CO. Red colors means emissions from CEDS are higher than that in MEIC, and blue colors means lower in CEDS. The relative differences are calculated as (CEDS-MEIC)/MEIC $\times 100$.
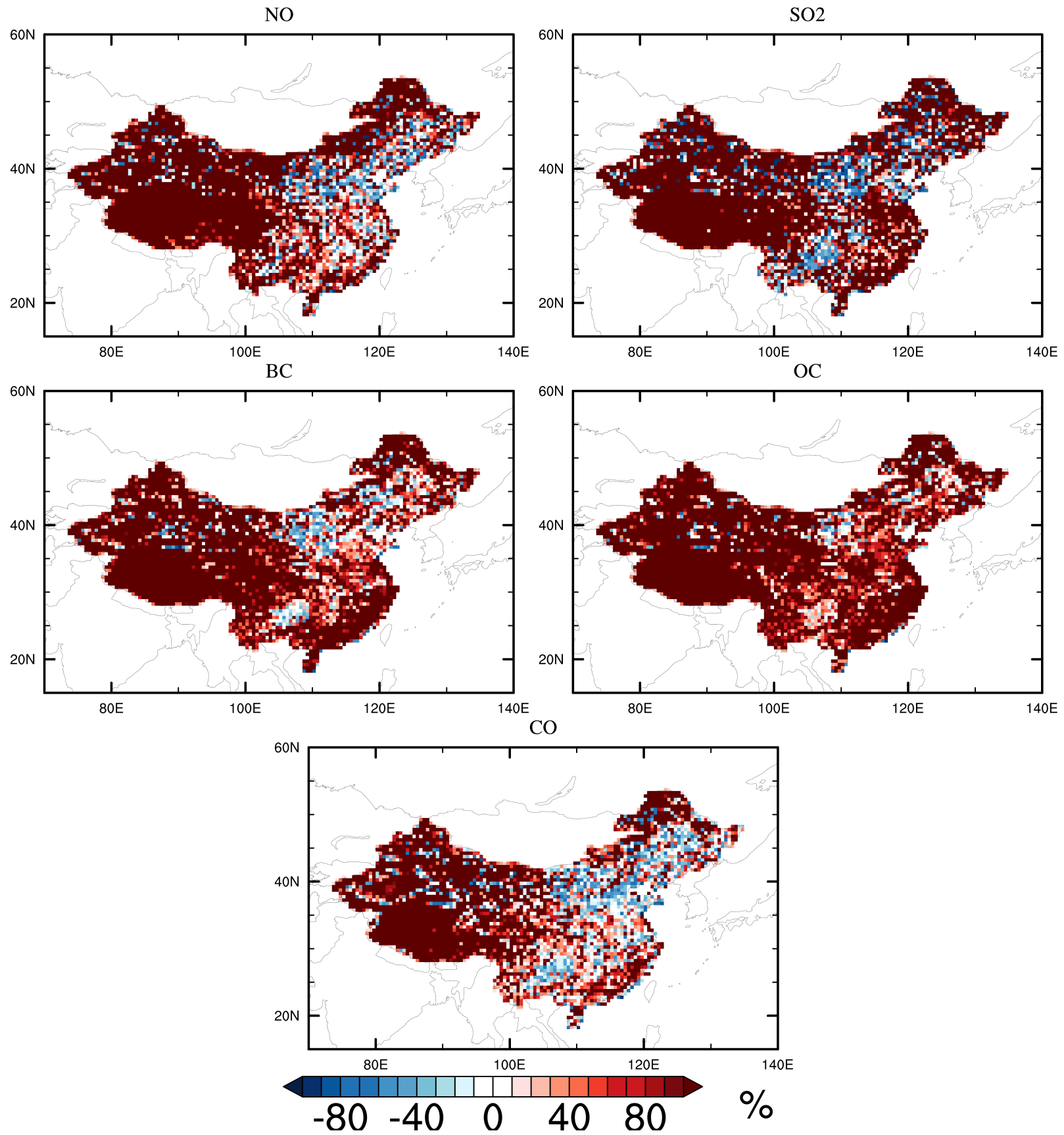
Fig. S3. Simulated annual $\mathrm{PM}_{2.5}$ concentration differences between applying CEDS and MEIC emission inventory in China from 2010 to 2014. The differences are calculated between CEDS_MEIC and CEDS_Global for each year.

CEDS - MEIC Diff in 2010 for Annual $\mathrm{PM}_{2.5}$
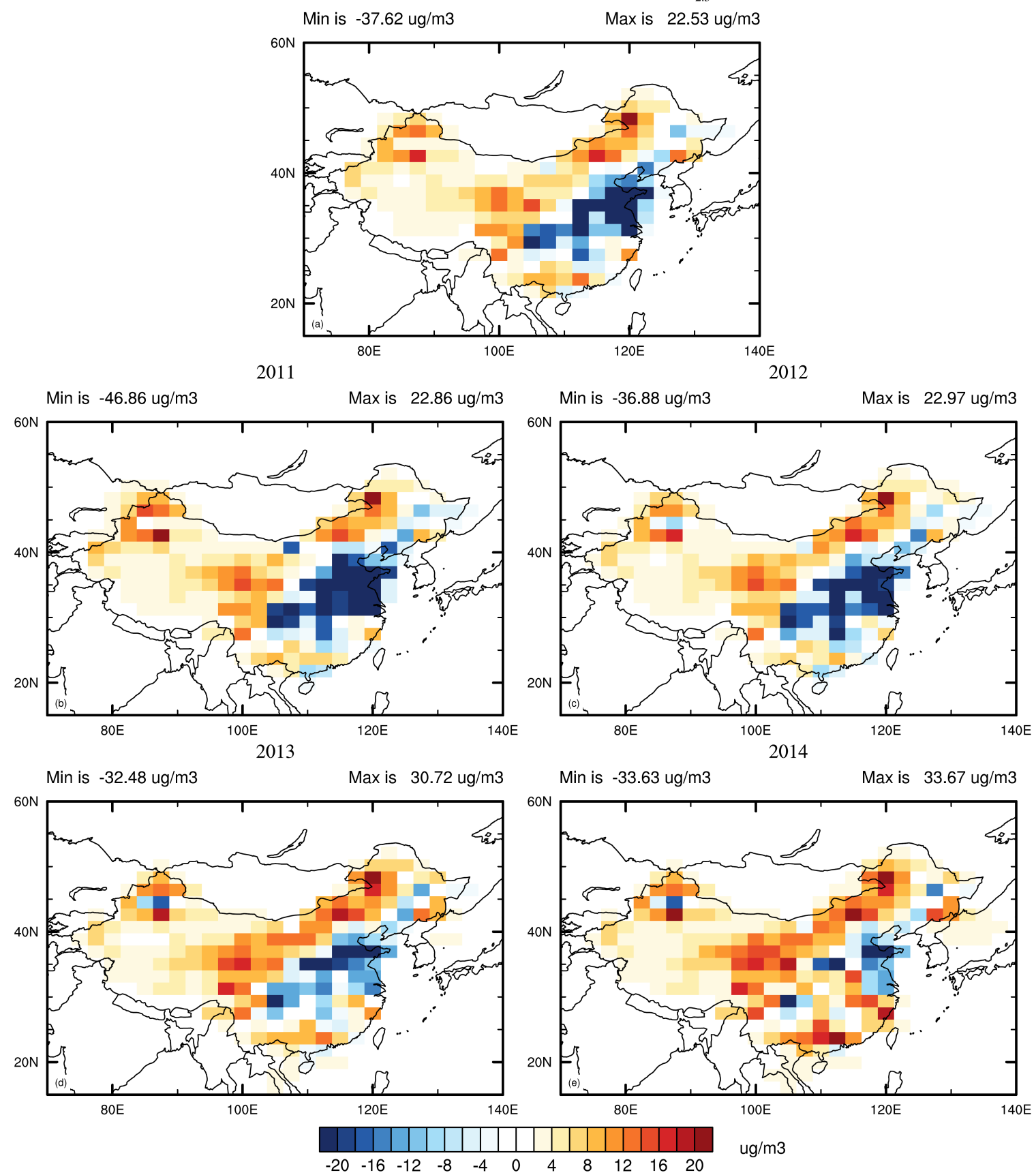
Fig. S4. As Fig. $\mathrm{S} 3$ but for annual average $\mathrm{MDA} 8 \mathrm{O}_{3}$ changes.

CEDS - MEIC Diff in 2010 for $\mathrm{O}_{3}$

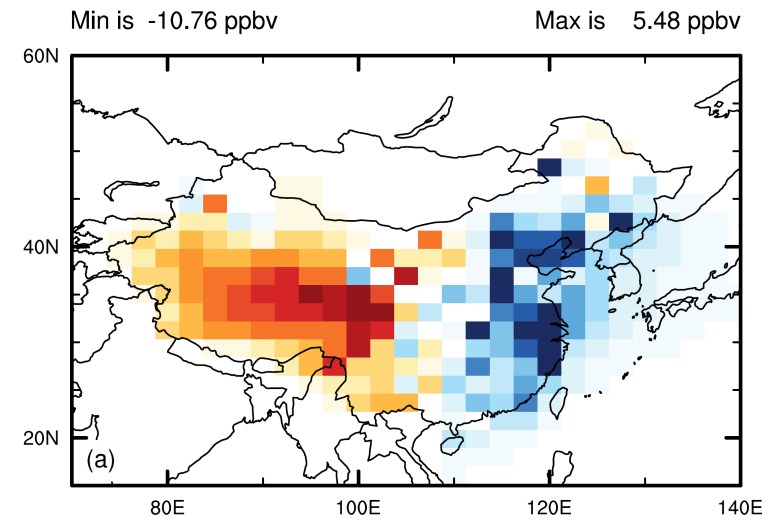

2011

Max is $6.20 \mathrm{ppbv} \quad$ Min is $-10.97 \mathrm{ppbv}$
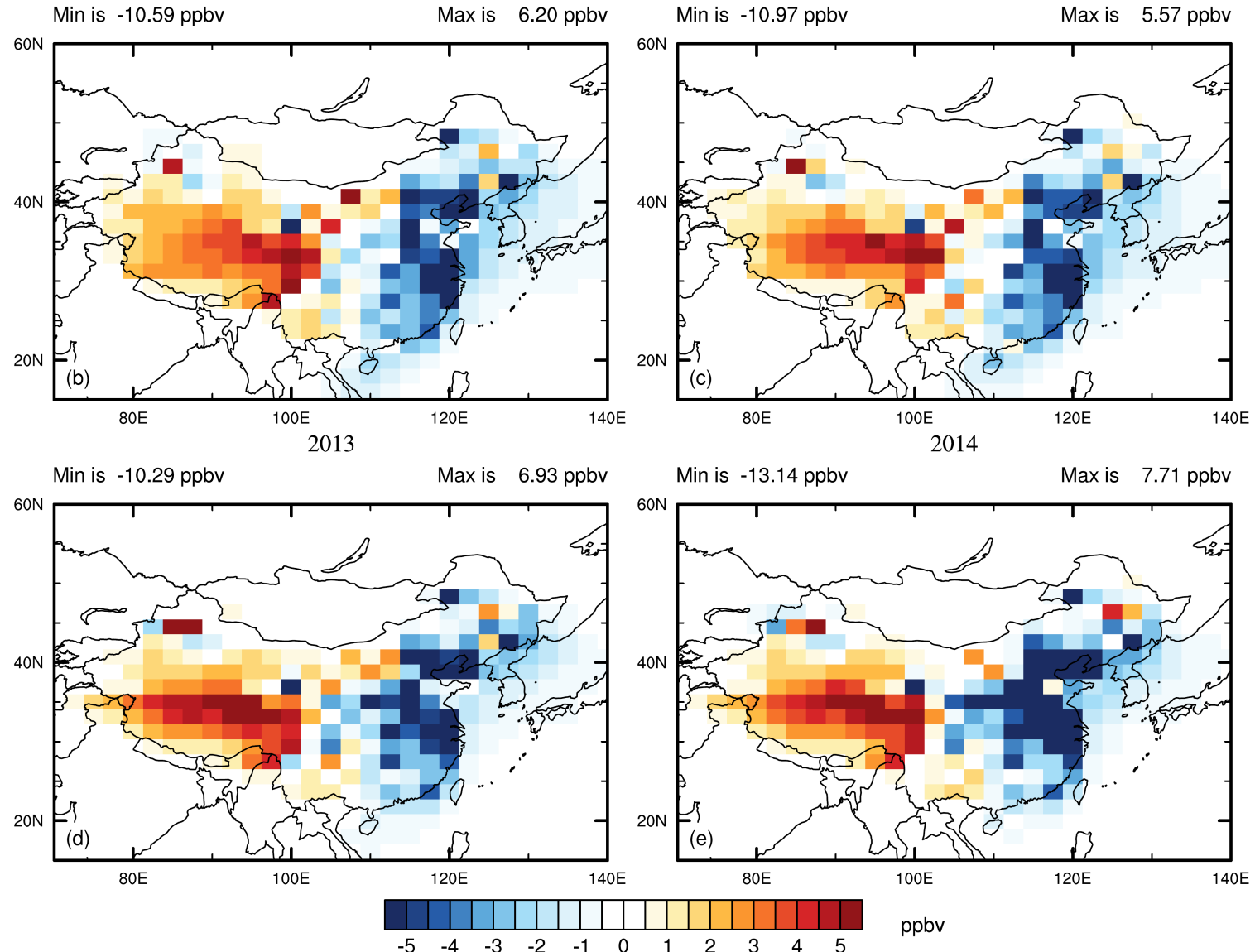
Fig. S5. Summertime MDA8 O3 changes from 2011 to 2017 from emission changes in China. The results are calculated as the differences between CEDS_MEIC and CEDS_MEIC_ChinaFix for each year.

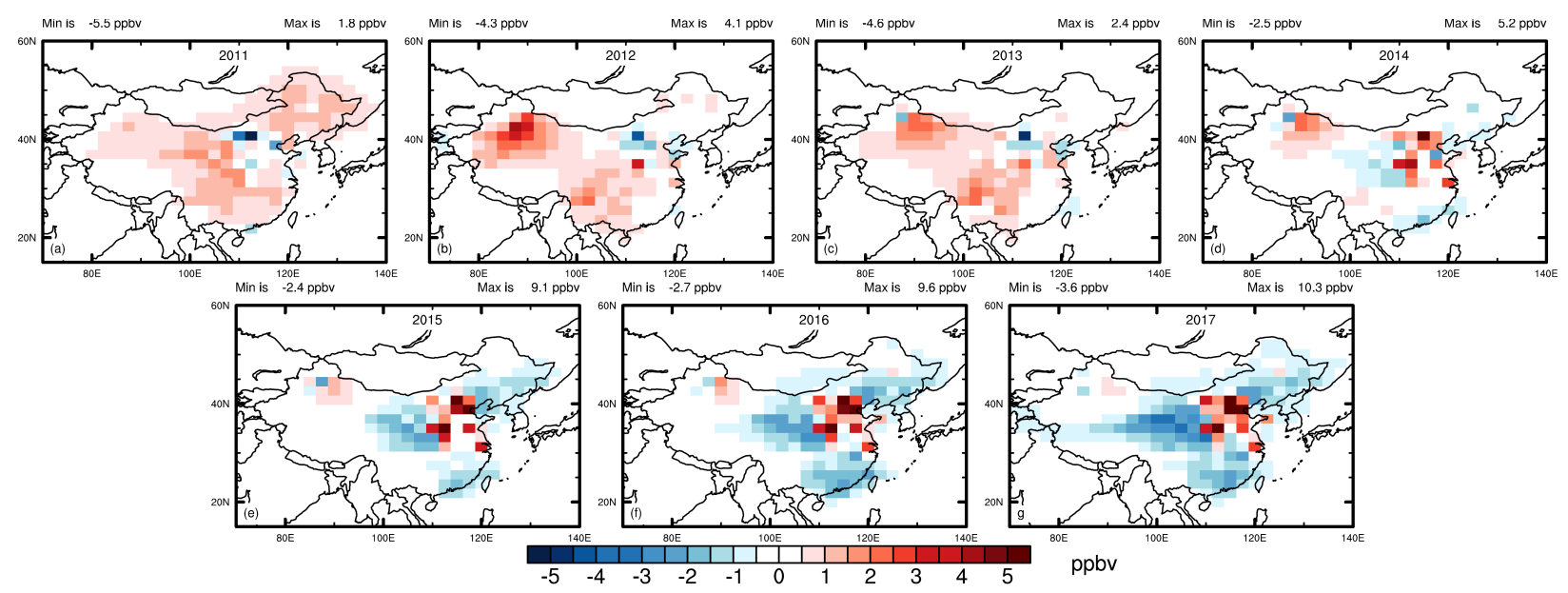

Fig. S6. Global zonal average $\mathrm{O}_{3}$ changes from 2010 to 2017 . The differences are calculated as the differences between each year (from 2011 to 2017) and 2010.

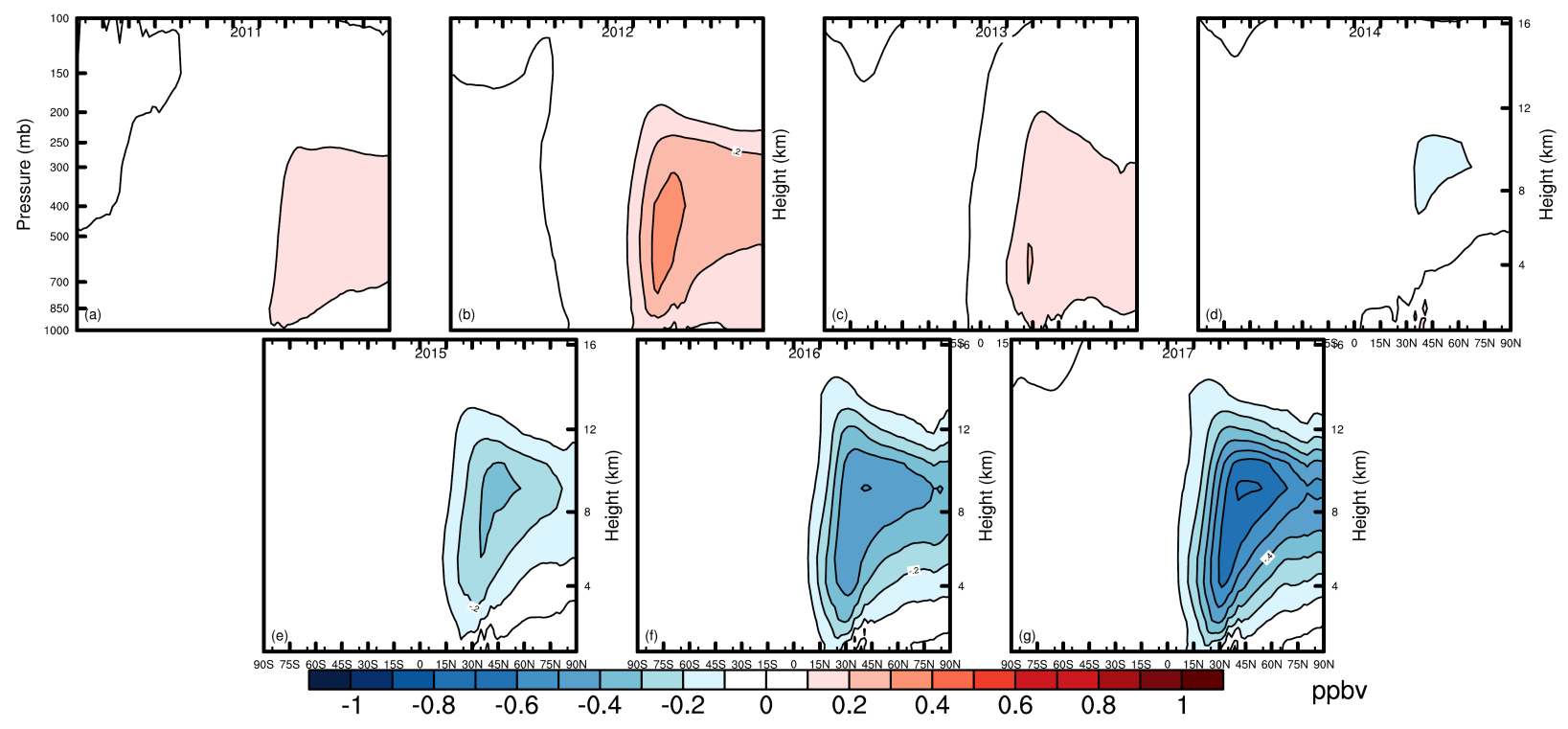

